\title{
Myofascial trigger points in migraine and tension-type headache
}

Thien Phu Do, Gerda Ferja Heldarskard, Lærke Tørring Kolding, Jeppe Hvedstrup and Henrik Winther Schytz ${ }^{*}$

\begin{abstract}
Background: A myofascial trigger point is defined as a hyperirritable spot in skeletal muscle that is associated with a hypersensitive palpable nodule in a taut band. It has been suggested that myofascial trigger points take part in chronic pain conditions including primary headache disorders. The aim of this narrative review is to present an overview of the current imaging modalities used for the detection of myofascial trigger points and to review studies of myofascial trigger points in migraine and tension-type headache.

Findings: Different modalities have been used to assess myofascial trigger points including ultrasound, microdialysis, electromyography, infrared thermography, and magnetic resonance imaging. Ultrasound is the most promising of these modalities and may be used to identify MTrPs if specific methods are used, but there is no precise description of a gold standard using these techniques, and they have yet to be evaluated in headache patients.

Active myofascial trigger points are prevalent in migraine patients. Manual palpation can trigger migraine attacks. All intervention studies aiming at trigger points are positive, but this needs to be further verified in placebo-controlled environments. These findings may imply a causal bottom-up association, but studies of migraine patients with comorbid fibromyalgia syndrome suggest otherwise. Whether myofascial trigger points contribute to an increased migraine burden in terms of frequency and intensity is unclear.

Active myofascial trigger points are prevalent in tension-type headache coherent with the hypothesis that peripheral mechanisms are involved in the pathophysiology of this headache disorder. Active myofascial trigger points in pericranial muscles in tension-type headache patients are correlated with generalized lower pain pressure thresholds indicating they may contribute to a central sensitization. However, the number of active myofascial trigger points is higher in adults compared with adolescents regardless of no significant association with headache parameters. This suggests myofascial trigger points are accumulated over time as a consequence of TTH rather than contributing to the pathophysiology.
\end{abstract}

Conclusions: Myofascial trigger points are prevalent in both migraine and tension-type headache, but the role they play in the pathophysiology of each disorder and to which degree is unclarified. In the future, ultrasound elastography may be an acceptable diagnostic test.

Keywords: Headache, Myofascial trigger point, Muscle, Treatment, Trigemino-cervical-complex, Migraine, Tension-type headache, Diagnostic test

\section{Background}

Migraine affects $16 \%$ of the population in Europe [1] with high individual and socioeconomic costs $[2,3]$. Several mechanisms have been proposed to be involved in its pathophysiology including vascular, peripheral and central mechanisms [4-9]. Jes Olesen systematically described pericranial tenderness in migraine patients, both

\footnotetext{
* Correspondence: henrik.winther.schytz.01@regionh.dk

Headache Diagnostic Laboratory, Danish Headache Center and Department of Neurology, Rigshospitalet Glostrup, Faculty of Health Sciences, University of Copenhagen, Glostrup, Denmark
}

during and outside of migraine attacks [10, 11], leading to speculations that myofascial mechanisms may be involved in migraine [12].

Tension-type headache (TTH) is the most prevalent primary headache disorder worldwide [13]. Tenderness in pericranial myofascial tissue is correlated with the intensity and frequency of headache in TTH [14-16], and studies show increased muscle stiffness in TTH patients $[17,18]$. Thus, myofascial structures may be associated with TTH pathophysiology. 
The term myofascial trigger point $(\mathrm{MTrP})$ was popularized in the 1950s and is defined as a hyperirritable spot in skeletal muscle that is associated with a hypersensitive palpable nodule in a taut band $[19,20]$. The spot is painful on compression and can cause referred pain, referred tenderness, motor dysfunction and autonomic phenomena. The interest in myofascial symptoms has been ongoing for centuries with similar descriptions of localized thickenings of muscles with regional pain [21]. There have been inconsistencies and controversies in the literature on the underlying pathology, and even the existence of MTrPs [22]. While attempts have been made to visualize MTrPs [22], the gold standard for detection of MTrPs has been unchanged since the 1950s [22] and remains to be by way of palpation of the affected muscles. However, this technique proves to be poorly reproducible as practitioners disagree on the location of MTrPs when blindly examining different patient groups [23]. Nevertheless, MTrPs have come to play a central role in the diagnosis and treatment of myofascial pain syndrome [19]. Furthermore, MTrPs have been proposed to take part in primary headache disorders and other chronic pain conditions [12]. The aim of this narrative review is to present an up-to-date overview on MTrPs in general and then in migraine and TTH, respectively.

\section{Review}

\section{Myofascial trigger points}

In the comprehensive trigger point manual by Travell and Simons [19], MTrPs are subclassified into different types, e.g., active and latent amongst others. An active MTrP produces a constant pain complaint while a latent only produces pain during manual palpation [19]. It was hypothesized that a sustained muscle contraction in MTrPs promotes hypoxia and ischemia with a following increase in concentrations of substances such as calcitonin gene-related peptide (CGRP) and substance P (SP) [24]. Consequently, this would lead to increased peripheral nociceptive transmission [24]. This hypothesis is only supported in active MTrPs, as they have been shown to be associated with higher levels of these substances in the local milieu compared to latent MTrPs $[25,26]$. Other properties such as the consistency of the tissue have also been suggested to play a key role in MTrPs [27].

\section{Investigations of myofascial trigger points Ultrasound imaging}

Different ultrasound modalities in ultrasound imaging have visualized MTrPs. Lewis et al. [28] conducted a pilot study to assess the use of ultrasound in determining soft tissue changes in the region of active MTrPs in 11 subjects. They found no correlation between clinical identified active MTrPs and ultrasound. In contrast, Turo et al. [29] were able to differentiate between symptomatic MTrPs and asymptomatic muscle tissue with texture-based analysis. Sikdar et al. investigated the stiffness of active and latent MTrPs, using ultrasound elastography by Doppler variance imaging in nine subjects while inducing vibrations with an external handheld massage vibrator [27]. MTrPs appeared as focal, hypoechoic regions on two-dimensional ultrasound images and with reduced vibration amplitude, indicating increased stiffness. Furthermore, they describe hypoechoic regions that were not identified during palpation prior to ultrasound. In another study by the same group, MTrPs showed reduced vibration amplitude on elastography indicating increased stiffness and distinct blood flow waveform patterns [30]. Ballyns et al. [31] used elastography to investigate MTrPs in 44 subjects with acute cervical pain. They were able to measure the size and distinguish type (active, latent) of MTrPs with elastography. In addition, Doppler waveforms of blood flow showed different characteristics in active sites compared to normal tissue. Takla et al. [32] compared elastography with two-dimensional grayscale ultrasound in identifying MTrPs. They found that MTrPs had an accuracy of $100 \%$ for both active and latent MTrPs while two-dimensional grayscale ultrasound could only identify 33 and $35 \%$, respectively.

\section{Microdialysis}

Microdialysis has been used to measure endogenous and exogenous molecules in the local milieu of MTrPs. Shah et al. [25] used microdialysis to investigate subjects with active or latent MTrP, and controls without MTrP were detected by manual palpation by two experienced clinicians. The authors measured selected substances $(\mathrm{pH}$, bradykinin (BK), CGRP, SP, tumor necrosis factor alpha (TNF- $\alpha$ ), interleukin 1 beta (IL-1 $\beta$ ), interleukin 6 (IL-6), interleukin 8 (IL-8), serotonin, and norepinephrine (NE)) in standardized locations of the trapezius muscle and gastrocnemius muscle. Subjects with active MTrPs in the trapezius muscle showed increased concentrations of all substances compared to the other groups. Shah et al. [26] found similar results in the trapezius muscle of subjects with neck pain and active MTrP compared to a group with neck pain and no MTrP present and healthy controls. The results showed that the active MTrP group had higher concentrations of BK, CGRP, SP, TNF- $\alpha$, IL-1 $\beta$, serotonin, NE.

\section{Electromyography}

Electromyography (EMG) can be used to measure the electrical activity of skeletal muscles. Simons et al. compared the prevalence of motor endplate potentials in active MTrPs, endplate zones, and taut bands of skeletal muscles in subjects with palpable MTrPs [33]. The authors found that endplate noise was more common in 
MTrPs than in sites outside of the trigger point, even within the same endplate zone. Ge et al. evaluated intramuscular muscle activity in a synergistic muscle during isometric contraction in 15 subjects with latent MTrPs [34]. The needle was inserted into a latent MTrP or a non-MTrP in the upper trapezius at rest and during contraction. The EMG activities were recorded from the middle deltoid muscle and the upper, middle, and lower parts of the trapezius muscle. The intramuscular EMG activity in the upper trapezius muscle was significantly higher at rest and during contraction at latent MTrPs compared with non-MTrPs. Yu et al. measured maximum voluntary isometric contraction, endurance, median frequency, and muscle fatigue index in three groups of participants: an active MTrP group, a latent MTrP group, and a control group [35]. The active MTrP group had a higher median frequency and muscle fatigue index than the control group. Wytrażek et al. compared the EMG activity of muscle motor units at rest and maximal contraction with surface EMG recordings [36]. The results showed MTrPs correlated with an increase in EMG amplitude at rest.

\section{Infrared thermography}

Infrared thermography can be used to measure the skin temperature. Dibai-Filho et al. [37] have reviewed the literature on infrared thermography investigations of MTrPs. The authors included three comparative studies [38-40] and one accuracy study [41]. The conclusion of the review is that the included studies do not agree on skin temperature patterns in the presence of MTrPs. The included studies of the review are briefly presented in the following. Merla et al. [38] found that individuals with myofascial pain had a greater difference between the right and left side in skin temperature over the masseter and sternocleidomastoid muscles before and after maximal voluntary clenching compared to healthy volunteers. They also found that the myofascial pain group had a greater temperature change over the measured muscles after maximum voluntary clenching. Kimura et al. [39] evaluated the vasoconstrictor response after provoking pain in MTrPs with an intramuscular glutamate injection. Furthermore, they activated the sympathetic outflow by using a breath-holding maneuver. They found a decrease in skin temperature over time in latent MTrPs. In contrast, Zhang et al. [40] did not find that the skin temperature was affected following an intramuscular glutamate injection into latent MTrPs. Haddad et al. [41] compared infrared thermography and algometer measurements of MTrPs in the masticatory muscles. The authors found a positive correlation between skin surface temperature and pressure pain threshold. Regarding diagnosing MTrPs, infrared thermography had an accuracy of 0.564 to 0.609 (area under the receiver operating characteristic curve).

\section{Magnetic resonance imaging}

Chen et al. [42] examined 65 patients with myofascial pain-associated taut bands using magnetic resonance elastography. They found that agreement between physicians and imaging raters were relatively poor $(63 \%$; $95 \%$ CI, 50\%-75\%), but that these bands could be assessed quantitatively using magnetic resonance elastography. The authors suggest that clinicians might overestimate while magnetic resonance elastography may underestimate MTrPs.

\section{Migraine and myofascial trigger points}

Pericranial tenderness in migraine patients was systematically described by Jes Olesen in 1978, both during and outside of attacks $[10,11]$ leading to speculations that myofascial mechanisms may be involved in migraine [12]. The bottom-up model states that increased peripheral nociceptive transmission sensitizes the central nervous system to lower the threshold for perceiving pain while the top-down model suggests these changes are already present in the central nervous system [43]. While it can be argued that pericranial tenderness in migraine may be caused by a top-down central sensitization, a bottom-up association was implied in 1981 when Tfelt-Hansen et al. [44] demonstrated that injections of lidocaine and saline into tender trigger points could relieve migraine attacks. They infiltrated the most tender spots of 26 cranial and neck muscles and tendon insertions in 50 migraine patients. The most frequent sites of tenderness were sternocleidomastoid, anterior temporal, neck and shoulder muscles, the coronoid process and occipital insertions. The tender points in the mentioned study do not necessarily overlap with Travell and Simons' definition of MTrPs, but the implication stands that peripheral myofascial mechanisms may be involved in migraine pathophysiology. Consequently, there has been an interest in exploring MTrPs in migraine (Table 1) [45-58].

\section{The occurrence of myofascial trigger points in migraine}

Several studies have demonstrated a high occurrence of active and latent MTrPs in migraine patients [45-49]. Studies show that there is a significantly higher prevalence of active MTrPs in migraine patients compared to healthy controls $[45,46,58]$. There are conflicting results in which muscles are the most affected [47, 48]. Fernández-de-Las-Peñas et al. [46] observed that active MTrPs were most prevalent ipsilateral to the migraine headaches. More unclear is whether the amount of MTrPs is correlated with the frequency and intensity of headache attacks. Calandre et al. [45] found a positive correlation between the number of MTrPs and 


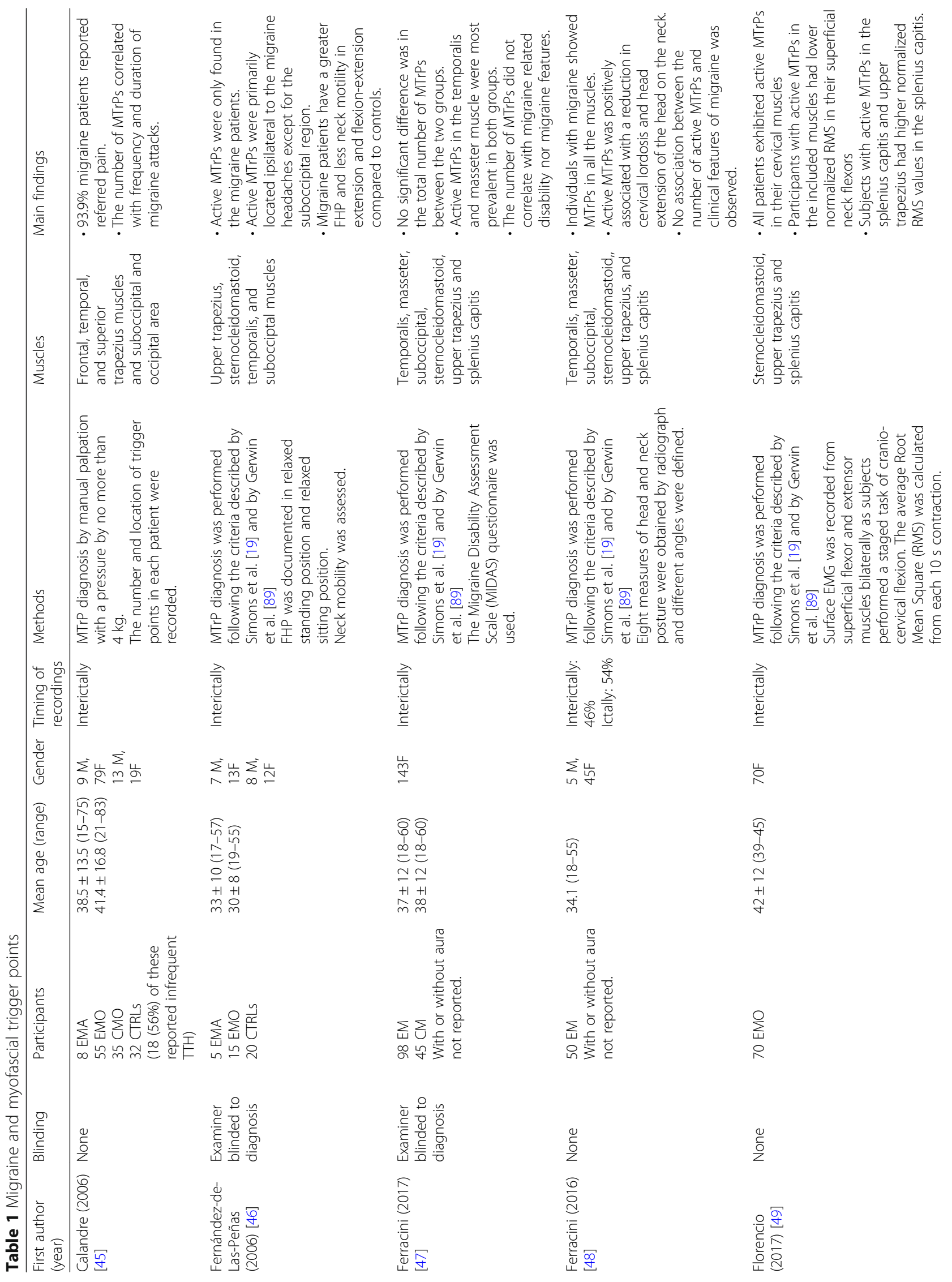




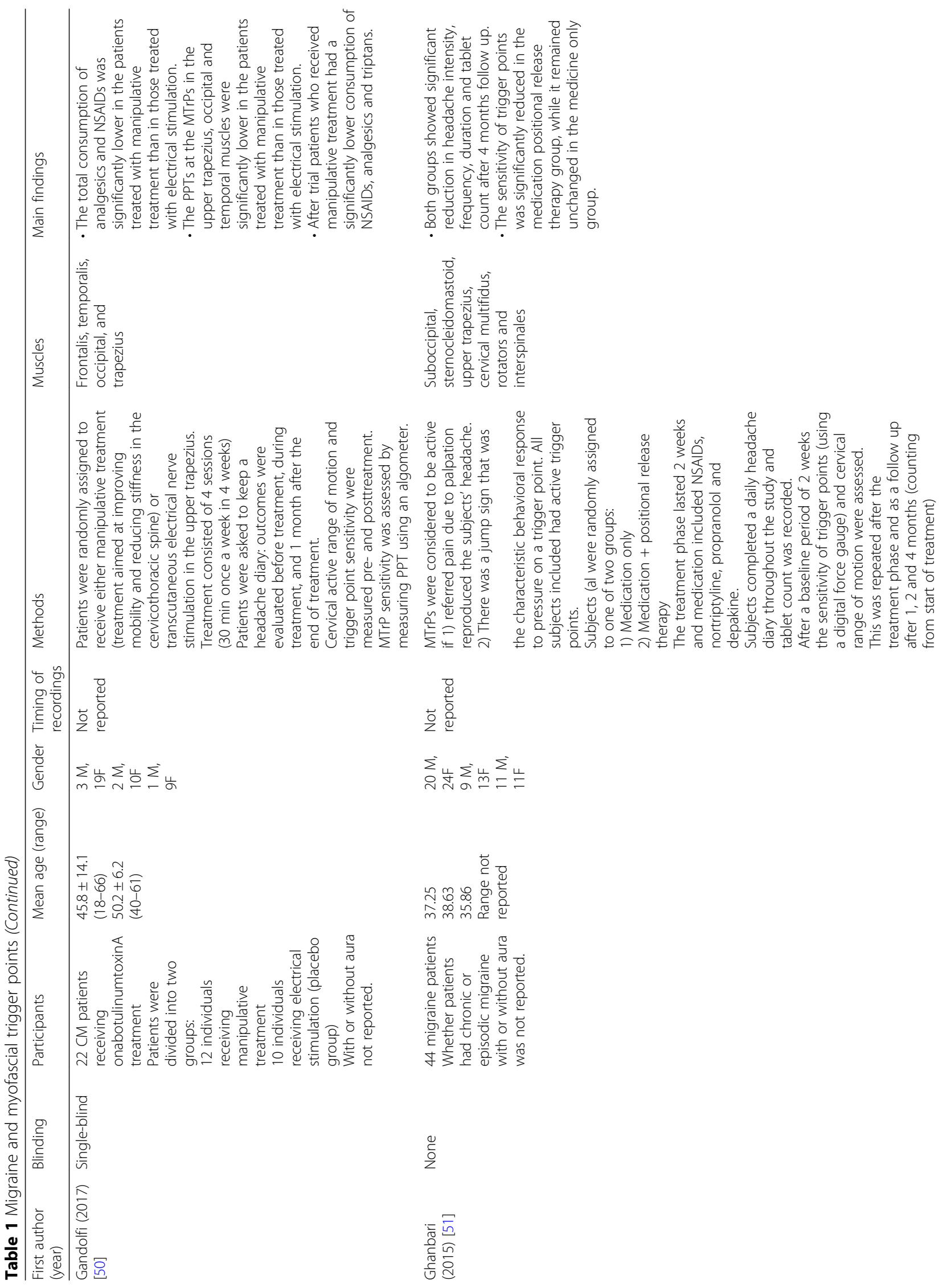




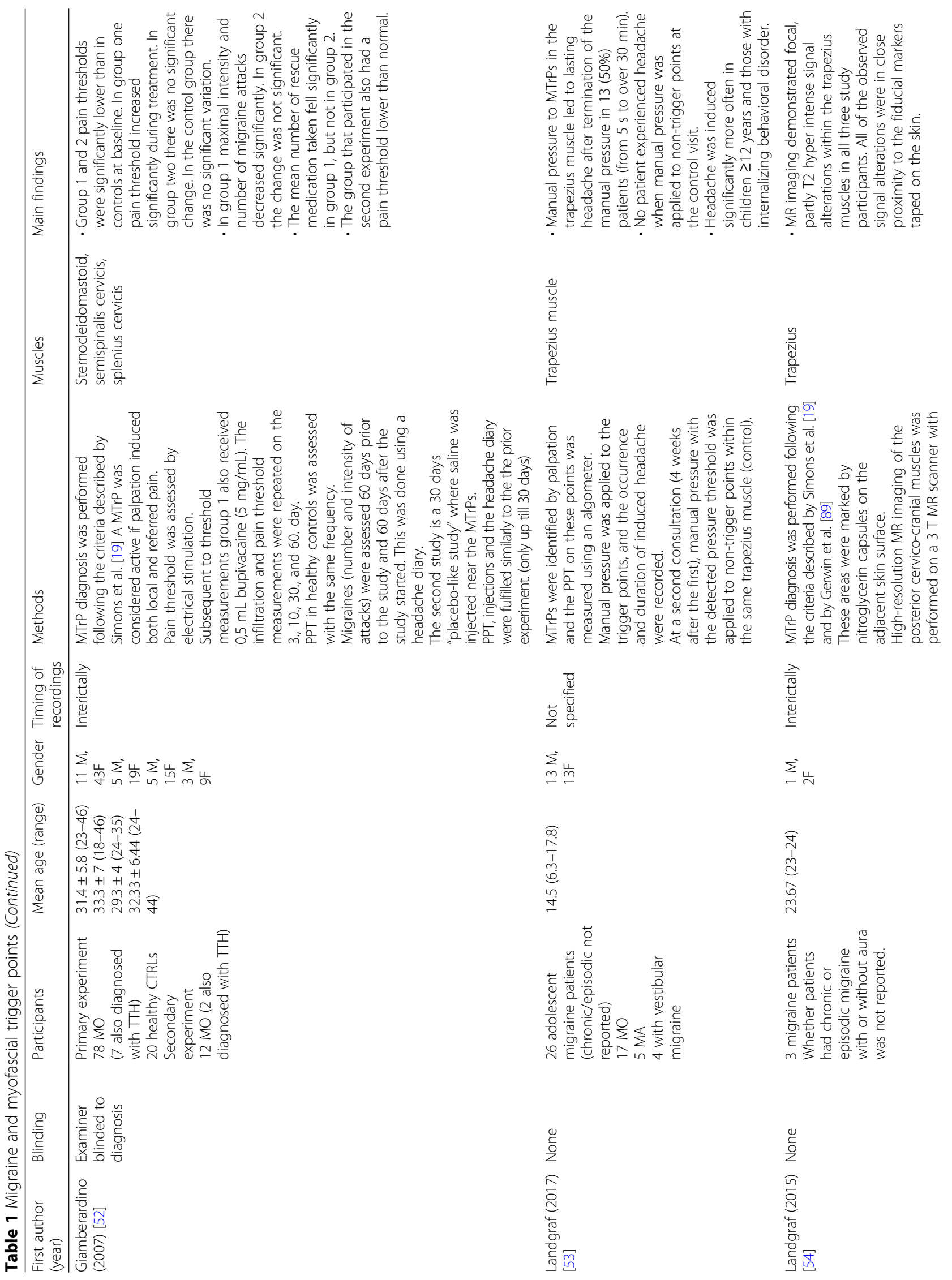




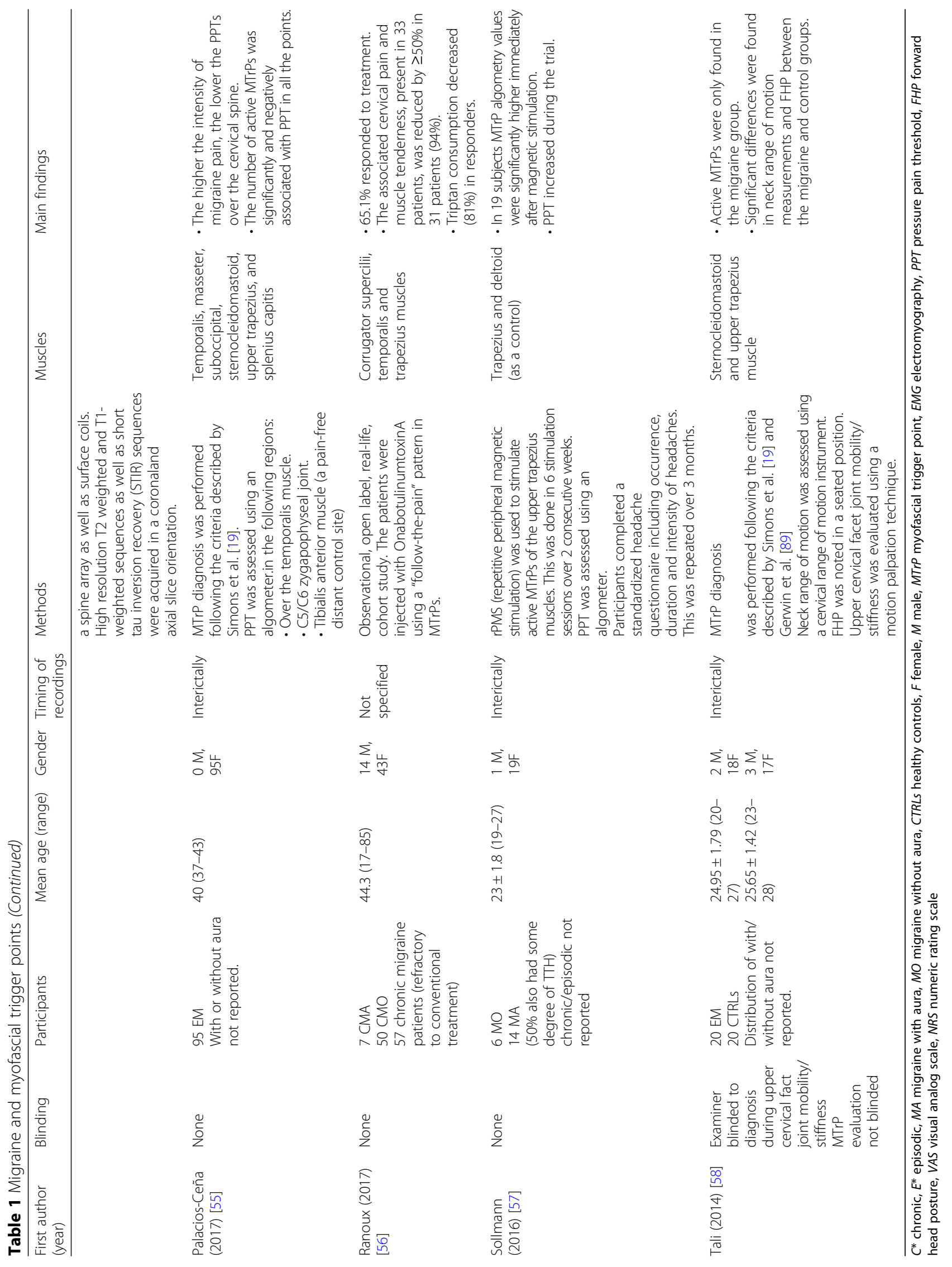


frequency and duration of migraine attacks, whereas two studies by Ferracini et al. [47, 48] found no such correlation. Interestingly, Landgraf et al. [54] could visualize MTrPs on MR imaging as focal signal alterations in a small pilot study.

\section{Neck mobility and specific muscles}

There appears to be an association between neck mobility and MTrPs [46, 48, 49, 58]. Ferracini et al. [48] found that a higher number of active MTrPs was positively correlated with a reduction in cervical lordosis and head extension of the head on the neck. In addition, that lower cervical angles were correlated higher then the number of active MTrPs. Florencio et al. [49] hypothesized that active MTrPs in the cervical musculature alters the activity of the related muscles and that this would be reflected in EMG readings. They observed that the presence of active MTrPs in the cervical musculature had different activation in the neck flexor muscles compared to those without active MTrPs in the same muscles regardless of the presence of pain. Palacios-Ceña et al. [55] found that the number of active MTrPs in head, neck and shoulder muscles were associated with widespread pressure hypersensitivity in a migraine population.

\section{Provocation and intervention studies}

Two unblinded studies show that manual palpation of MTrPs can provoke a migraine attack $[45,53]$. Calandre et al. provoked a migraine attack in one-third of a migraine population by palpating MTrPs [45]. Landgraf et al. provoked migraine headache by inducing pressure to MTrPs and could not replicate this by pressure to non-trigger points in the trapezius in an adolescent migraine population [53].

Interventions targeted at MTrPs show promising results [50-52, 56, 57], but the quality of studies varies greatly and lack placebo-control. Giambierardino et al. demonstrated that local anesthetic infiltration of MTrPs resulted in a reduction of migraine symptomatology in terms of frequency and intensity [52]. Furthermore, there was a reduction of hyperalgesia, not only at the injection site but also in referred areas overlapping with migraine pain sites. Similar, Ranoux et al. injected botulinum toxin in MTrPs with similar results in terms of reduction in headache days [56]. Gandolfi et al. improved the outcome of prophylactic botulinum toxin treatment in chronic migraine patients with manipulative treatment of MTrPs [50]. The outcome was a lower consumption of analgesics, improvement in pressure pain threshold and increased cervical range of motion. Likewise, Ghanbari et al. reported that combined positional release therapy targeted at MTrPs with medical therapy is more effective than the sole pharmacological treatment [51]. Interestingly, sessions of magnetic stimulation of active MTrPs reduced headache frequency and intensity in adolescent migraineurs [57]. Though these findings need to be verified in a placebo-controlled study. There has not been any studies on the effect of systemic musculoskeletal analgesics on MTrPs [59], which would be of interest for future studies.

\section{Tension-type headache and myofascial trigger points}

Both peripheral and central mechanisms have been suggested as important components of TTH [14-16, 60]. Tenderness in pericranial myofascial tissue is correlated with the intensity and frequency of headache [14-16]. Furthermore, there has been demonstrated increased muscle stiffness in the trapezius muscle in TTH patients $[17,18]$ not differing between headache and non-headache days [18]. Although a recent study found no increased muscle stiffness in TTH patients, this may be due to the method used [61]. Studies show that the referred pain elicited by active MTrPs reproduce the headache pattern in TTH patients [62-66]. Accordingly, there has been an interest in investigating the occurrence of MTrPs in TTH (Table 2) [62-80].

\section{The occurrence of myofascial trigger points in tension-type headache}

There is a high occurrence of active and latent MTrPs in patients with TTH $[63-67,69-72,79]$ Active MTrPs are found almost only in TTH patients compared to controls $[63,65,69,72,80]$. MTrPs are more prevalent on the dominant side of the patients [66]. The number of active MTrPs is higher in adults in comparison to adolescents regardless of no significant association between the number of active MTrPs and headache frequency, duration and intensity [62]. Other studies have found that active MTrPs are correlated with the severity of TTH $[65,67,78,80]$ with a greater occurrence of MTrPs in chronic TTH in comparison to episodic TTH [80]. Furthermore, studies show that active MTrPs are correlated with the intensity, duration and frequency of headache episodes in TTH $[65,80]$. In contrast, other studies failed to show a correlation between MTrPs and chronic and frequent episodic TTH [78] and showed no correlation between MTrPs and headache parameters either in episodic TTH patients [69].

\section{Neck mobility and specific muscles}

Episodic TTH patients had less neck mobility compared to controls [69]. Patients with active MTrPs had a greater forward head position than subjects only with latent MTrPs [69]. However, neither forward head position or neck mobility was correlated with headache parameters [69]. In a different study, active MTrPs in the right upper trapezius muscle and left sternocleidomastoid muscle was correlated with a greater headache intensity and duration [72]. Furthermore, active MTrPs in the right and left 


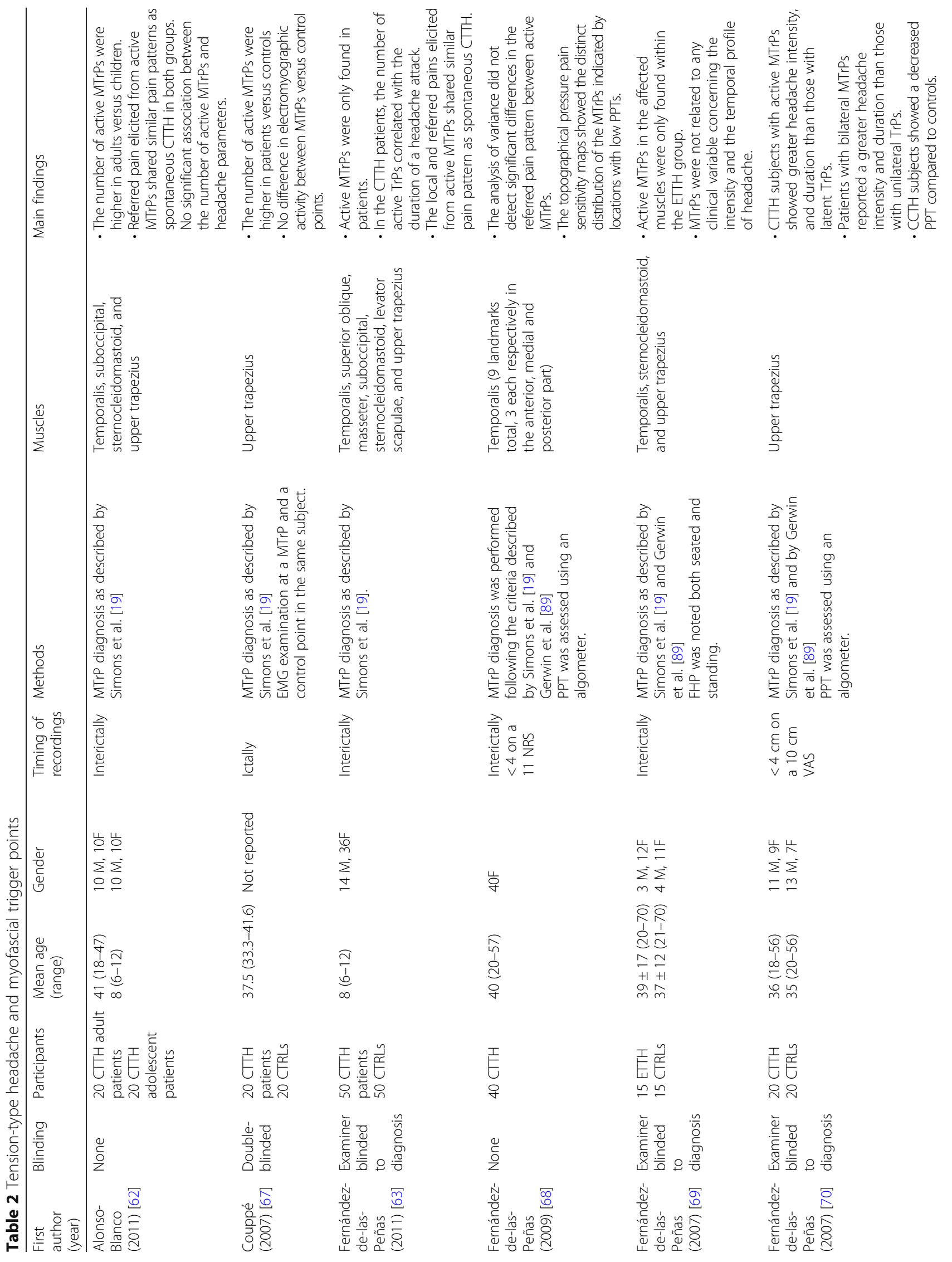




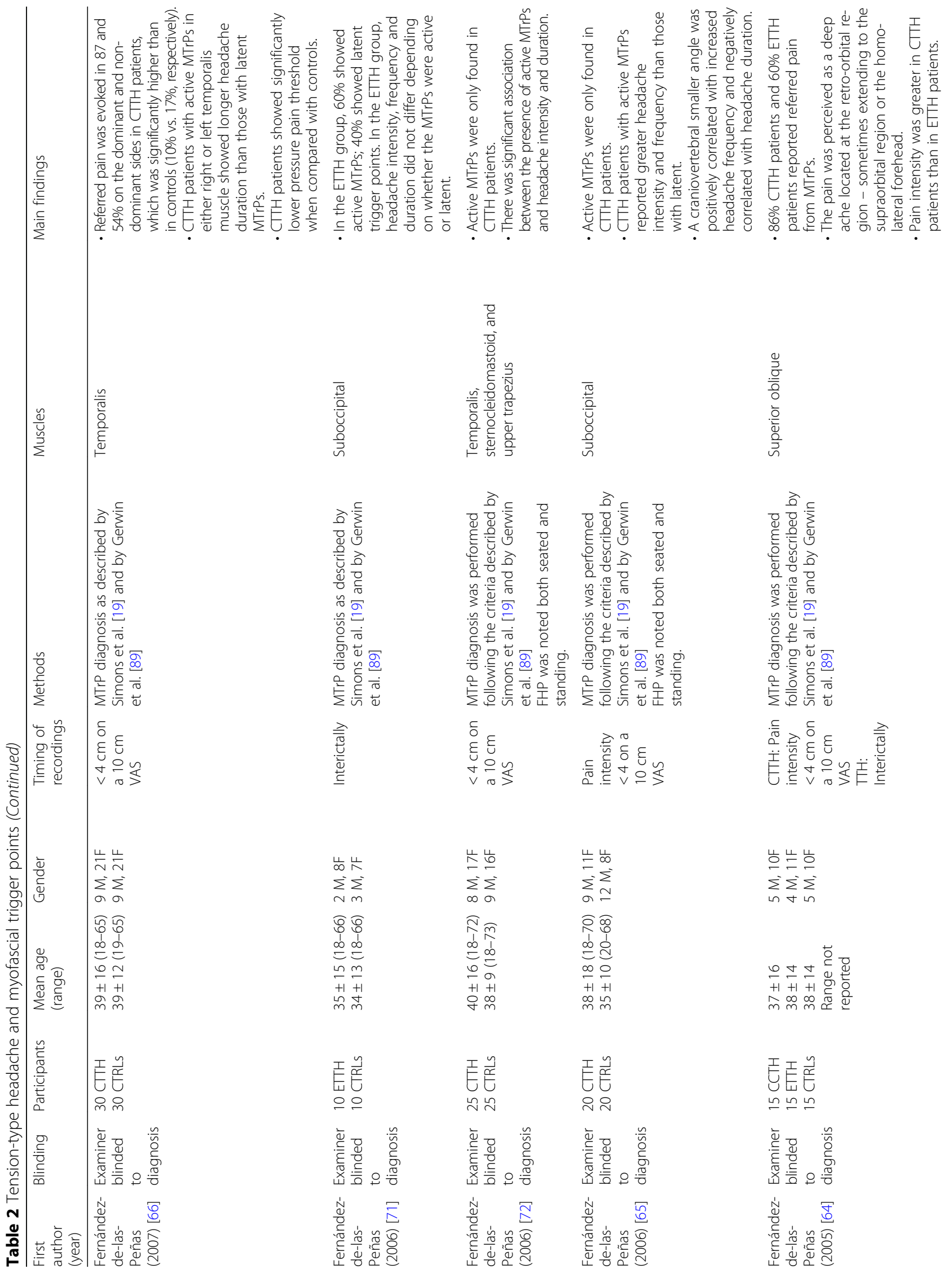




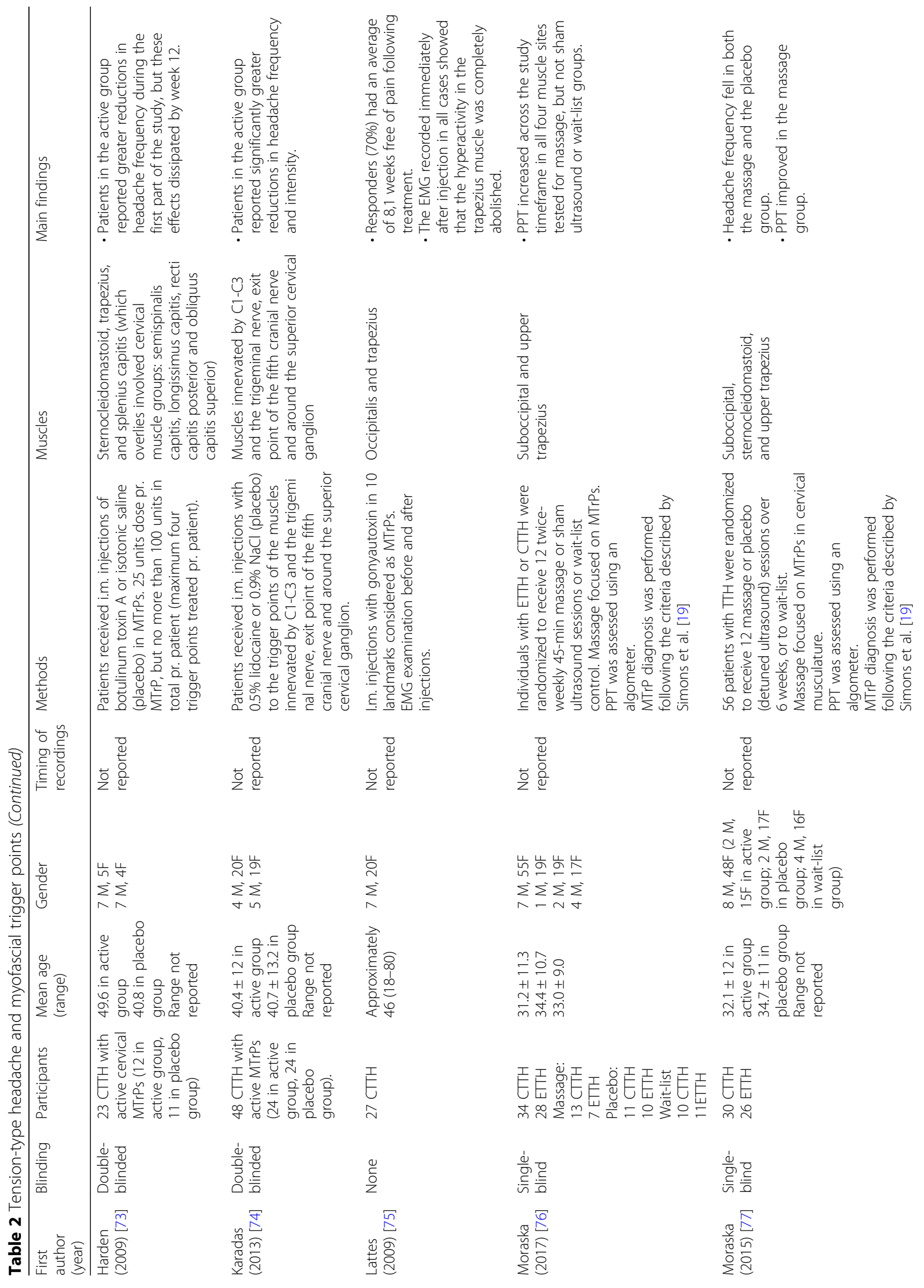


Do et al. The Journal of Headache and Pain (2018) 19:84

Page 12 of 17

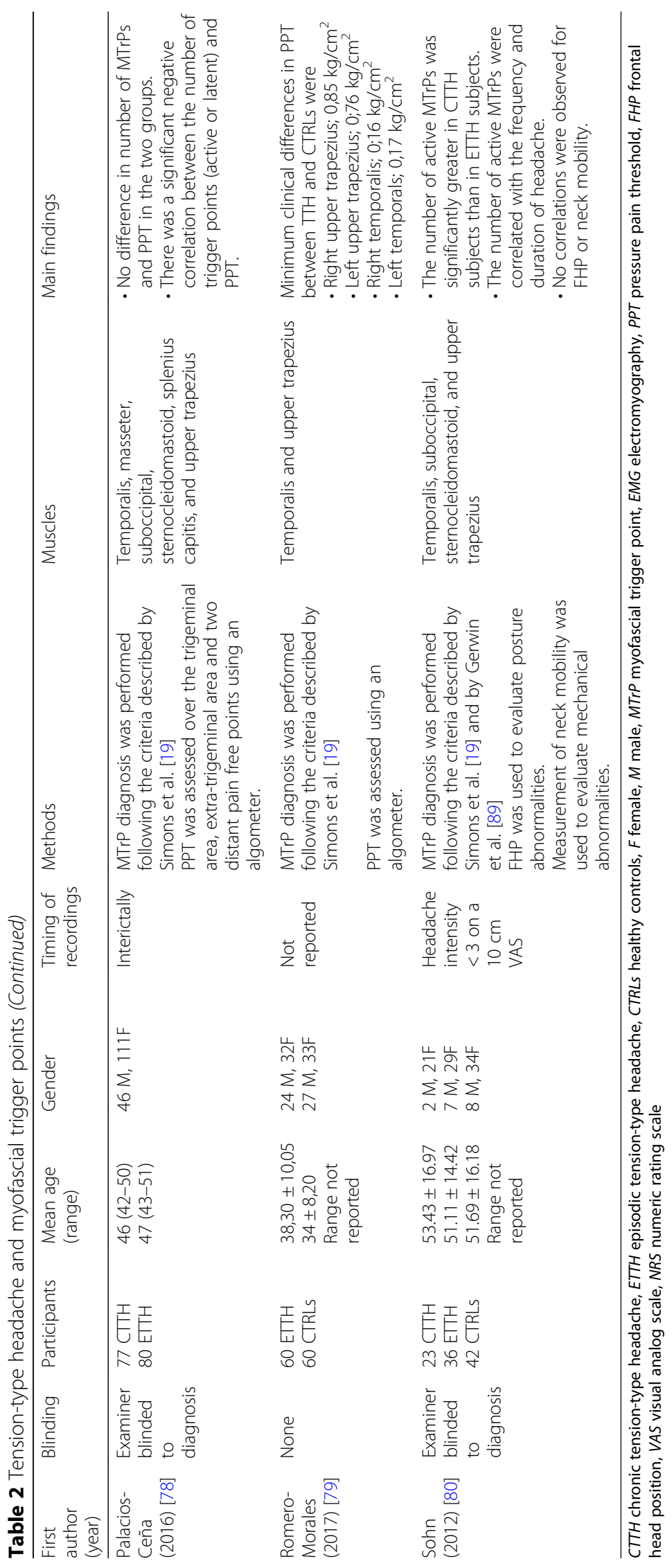


temporalis muscles correlated with longer headache duration and greater headache intensity, respectively [72]. Suboccipital active MTrPs correlated with increased intensity and frequency of headache [65]. Chronic TTH patients with active MTrPs in the analyzed muscles had a greater forward head position than those subjects only with latent MTrPs [65, 72]. Sohn et al. [80] identified a greater occurrence of MTrPs in chronic TTH compared to episodic TTH and that the number of active MTrPs correlated with the frequency and duration of headache, although they did not find any correlations for forward head posture and neck mobility in contrast to Fernández-de-las-Peñas et al. [65, 72].

\section{Pressure pain threshold}

The number of active and latent MTrPs was significantly and negatively associated with pressure pain thresholds on the temporalis muscle, C5/C6 zygapophyseal joint, second metacarpal, and tibialis anterior muscle [78]. Thus, a higher number was associated with a more generalized sensitization regardless of the frequency of headache. Another study observed that the location of active MTrPs in the temporalis muscle corresponded to areas with lower pain pressure thresholds which establishes a relationship between the two [68]. The same group found that chronic TTH patients with bilateral active MTrPs in the trapezius muscles have a significantly lower pain pressure threshold compared to patients with only unilateral active MTrPs [70]. Minimum clinical differences in pressure pain thresholds in TTH patients may be used to evaluate treatment of MTrPs [79].

\section{Therapeutic studies targeting myofascial trigger points}

Karadas et al. [81] investigated pericranial lidocaine injections in MTrPs in 108 patients with frequent episodic TTH using a double-blind placebo-controlled randomized study design. Repeated local lidocaine injections into the MTrPs in the pericranial muscles reduced both the frequency and intensity of pain compared to placebo. Another placebo-controlled study found similar results with lidocaine injections in MTrPs in chronic TTH with a reduction in pain frequency, pain intensity, and analgesic use [74]. In addition, there was a significant effect on anxiety and depression of the subjects. A randomized, double-blind, placebo-controlled pilot study of botulinum toxin A injections in MTrPs included 23 patients with chronic TTH [73]. The subjects were assessed at 2 weeks, 1, 2 and 3 months after injection. The botulinum toxin A group reported a reduction in headache frequency that disappeared by week 12 . There was no difference in intensity between the groups. In a randomized, placebo-controlled clinical trial, Moraska et al. applied massage focused on MTrPs of patients with $\mathrm{TTH}$
[77]. For both active and placebo groups, there was a decrease in headache frequency, but not for intensity or duration. Thus, there was no difference between massage and placebo [81].

\section{Discussion}

Ultrasound and EMG appear to be the most promising modalities to be used as a diagnostic test for MTrPs. While the use of ultrasound in headache disorders has primarily been focused on vascular changes and not on myofascial structures [82], ultrasound may also be used to identify MTrPs if specific analysis methods are applied [29] or with the use of elastography [27, 30, 32]. However, there is no precise description of a gold standard using these techniques, and they have yet to be evaluated in headache patients. Active MTrPs affect the electrical activity at rest and during muscle contraction in EMG studies [33-36]. Out of the two modalities, ultrasound is presumably the most viable candidate as a diagnostic test as it has an immediate availability at most treatment sites, it is time-efficient and is non-invasive. Although there are currently no studies investigating if it is possible to identify MTrPs with ultrasound without prior manual palpation. Future studies should investigate if ultrasound is comparable with manual palpation in identifying MTrPs. The other modalities do not appear to be suitable as microdialysis show mixed results regarding whether the local milieu of MTrPs is changed and needs further exploration before a conclusion can be made $[25,26,83]$. According to the review by Dibai-Filho et al. [37], infrared thermography appears to be a promising non-invasive method, but it should still only be used as an auxiliary tool in the evaluation of MTrPs due to conflicting results. Magnetic resonance elastography in diagnosing MTrPs has only been investigated in a few studies, and the sensitivity may be too low for suitable use as a diagnostic test [42].

Studies show a high occurrence of active and latent MTrPs [45-49] and correlation between neck mobility and MTrPs in migraine patients $[46,48,49,58]$. However, there are conflicting results in which muscles are the most affected [47, 48], and it is unclear whether there is a positive correlation with the degree of headache frequency or intensity due to conflicting results. Palpation of MTrPs may provoke a migraine attack in some patients $[45,53]$ but needs further confirmation in placebo-controlled studies. Intervention studies targeting MTrPs are mostly positive [50-52, 56, 57], but they lack placebo-control. Thus, a bottom-up association between MTrPS and migraine [44] cannot be fully supported based on the evidence (Fig. 1). In addition, in patients with migraine-fibromyalgia comorbidity, it 


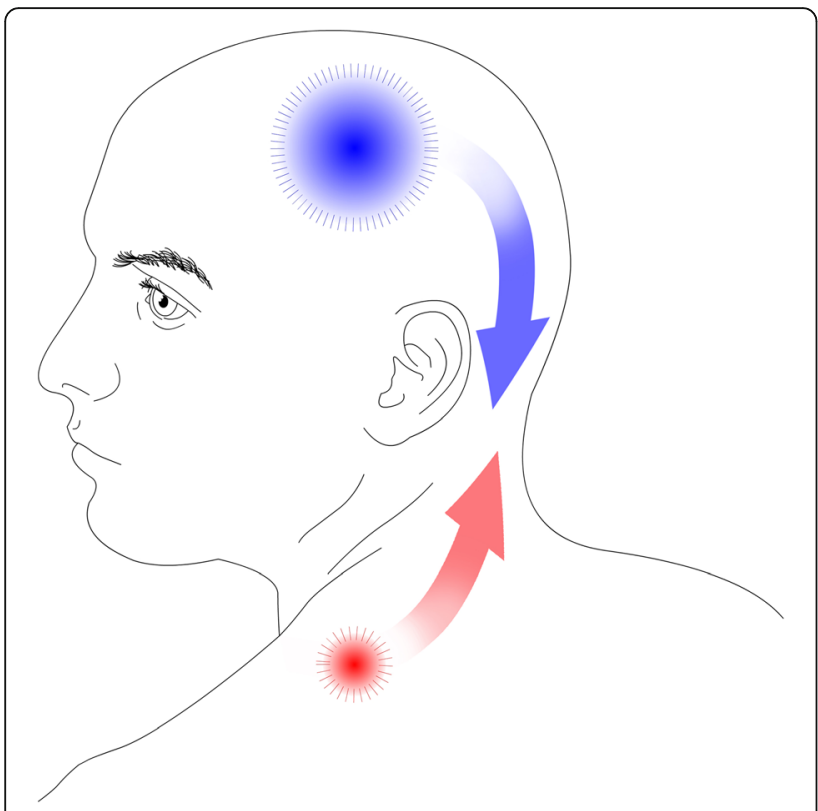

Fig. 1 The bottom-up model states that increased peripheral nociceptive transmission sensitizes the central nervous system to lower the threshold for perceiving pain while the top-down model suggests these changes are already present in central nervous system. In relation to myofascial trigger points, a bottom-up model would suggest that increased nociceptive transmission from myofascial trigger points lowers the threshold for perceiving pain (red). A top-down model would suggest that central sensitization may contribute to the occurrence of myofascial trigger points rather than the other way around (blue)

has been shown that migraine attacks exacerbate fibromyalgia symptoms, suggesting a top-down central sensitization [84] as fibromyalgia symptoms include specific tender points [85]. Although a study showed migraine severity was similar in migraine patients with and without fibromyalgia [86]. One would expect an association between migraine severity and co-existing fibromyalgia if a top-down central is taking place in patients with this comorbidity. It is possible that MTrPs may have an important role in some subpopulations of migraine patients. This calls for therapeutic studies targeting patients with a high degree of MTrPs, but this is only speculative at this point.

The prevalence of active MTrPs in TTH [65-67, 73, $74,77,78,80,81]$ is coherent with the hypothesis that peripheral mechanisms are involved in the pathophysiology of TTH $[14-16,60]$. It has been speculated that an increased peripheral nociception increases the sensitization of central mechanisms resulting in an increase in the sensitivity to peripheral pain (Fig. 1). Active MTrPs may contribute to a central sensitization as they are correlated with lower pain pressure thresholds $[68,70,78]$. This would also provide an explanation for the efficacy of injections of lidocaine in MTrPs [74, 81] as these would reduce the transmission of peripheral nociception. However, these assumptions are in contrast with a study showing that the number of active MTrPs is higher in adults in comparison to adolescents, regardless of no significant association with headache parameters [62]. This suggests that active MTrPs are accumulated over time as a consequence of TTH [62] instead of being an integrated part of the pathophysiology of TTH. Previous studies of botulinum toxin $\mathrm{A}$ injections in pericranial muscles have been shown to have no effect in TTH [87]. The efficacy of botulinum toxin A in MTrPs [73] might be explained by its possible action of modulating the release of nociceptive and inflammatory mediators e.g., CGRP and SP [88]. These inflammatory mediators may be increased in the local milieu of MTrPs [25, 26]. This would also account for its poor efficacy in injection protocols targeting fixed landmarks in pericranial muscles instead of MTrPs [87], as these substances appear to be concentrated at MTrPs $[25,26]$.

There are many overlapping findings in studies of MTrPs in migraine or TTH. In both disorders, MTrPs are prevalent and may be related to neck mobility. Palpation of MTrPs can, in some cases, provoke an attack in migraine patients, while palpation of MTrPs in TTH can provoke pain resembling the usual headache pattern of patients. Intervention studies are promising in both disorders. The quality of studies in both disorders varies greatly as many of the reviewed studies lacked blinding (Table 3). Furthermore, true blinding is difficult to achieve as active MTrPs by definition cause referred pain.

\section{Conclusion}

In conclusion, ultrasound elastography is the most promising tool to assess MTrPs [27, 30, 32], but still needs to be performed combined with palpation, which introduces risk of bias and interobserver variation. MTrPs are very frequent in both migraine patients [45-49] and TTH patients [65-67, 73, 74, 77, $78,80,81]$ compared to healthy controls. Active

Table 3 An overview on the use of blinding, control groups and placebo

\begin{tabular}{llll}
\hline & Migraine & Tension-type headache & \\
\hline Blinding & $36 \%(5 / 14$ relevant studies) & $79 \%(15 / 19$ relevant studies) & $61 \%(19 / 33$ relevant studies) \\
Control group & $44 \%(4 / 9$ relevant studies) & $79 \%(11 / 14$ relevant studies) & $65 \%(15 / 23$ relevant studies) \\
Placebo & $40 \%(2 / 5$ relevant studies) & $80 \%(4 / 5$ relevant studies) & $60 \%(6 / 10$ relevant studies) \\
\hline
\end{tabular}


MTrPs are especially interesting as these are rarely found in control groups. However, their role in the pathophysiology of each disorder and to which degree is still unclear. The results of the provocation and intervention studies support the hypothesis of a trigemino-cervical-complex pathophysiology model in both migraine $[45,50-53,56,57]$ and TTH $[73,74,81]$. Whether MTrPs contribute to an increased disease burden in migraine is uncertain $[45,47,48]$ and needs further exploration [50, 52]. Future research should aim to increase the quality of studies before further speculations are made. To elucidate this, large-scale studies to stratify the headache populations into more homogenous subgroups should be conducted.

\section{Abbreviations \\ BK: Bradykinin; CGRP: Calcitonin gene-related peptide; CTRL: Healthy control; EMG: Electromyography; F: Female; FHP: Forward head posture; IL-1 1 : Interleukin 1 beta; IL-6: Interleukin 6; IL-8: Interleukin 8; M: Male; MA: Migraine with aura; MO: Migraine without aura; MTrP: Myofascial trigger point; NE: Norepinephrine; NRS: Numeric rating scale; PPT: Pressure pain threshold; SP: Substance P; TNF-a: Tumor necrosis factor alpha; TTH: Tension-type headache; VAS: Visual analog scale \\ Funding \\ TPD and JH were funded by a grant from Candys Foundation. LTK was funded by a grant from the Lundbeck Foundation.}

\section{Availability of data and materials}

Data sharing is not applicable to this article as no datasets were generated or analysed during the current study.

\section{Authors' contributions}

TPD contributed with data interpretation, drafting and revision of the manuscript for intellectual content. GFH, LTK and JH contributed with revision of the manuscript for intellectual content. HWS contributed with conceptualization, data interpretation and revision of the manuscript for intellectual content. All authors read and approved the final manuscript.

\section{Ethics approval and consent to participate}

Not applicable.

\section{Consent for publication}

Not applicable.

\section{Competing interests}

HWS has received travel grants or speaking fees from Pfizer, Autonomic Technologies and Novartis. TPD, GFH, LTK and JH declare that they have no competing interests.

\section{Publisher's Note}

Springer Nature remains neutral with regard to jurisdictional claims in published maps and institutional affiliations.

Received: 21 August 2018 Accepted: 3 September 2018 Published online: 10 September 2018

\section{References}

1. Stovner LI, Andree C (2010) Prevalence of headache in Europe: a review for the Eurolight project. J Headache Pain. 11:289-299

2. Lyngberg AC, Rasmussen BK, Jørgensen T et al (2005) Secular changes in health care utilization and work absence for migraine and tension-type headache: a population based study. Eur J Epidemiol 20:1007-1014

3. Olesen J, Sobscki P, Truelsen T et al (2008) Cost of disorders of the brain in Denmark. Nord J Psychiatry 62:114-120
4. Olesen J, Burstein R, Ashina M et al (2009) Origin of pain in migraine: evidence for peripheral sensitisation. Lancet Neurol 8:679-690

5. Noseda R, Burstein R (2013) Migraine pathophysiology: anatomy of the trigeminovascular pathway and associated neurological symptoms, CSD, sensitization and modulation of pain. Pain 154:44-53

6. Shevel $E$ (2011) The extracranial vascular theory of migraine--a great story confirmed by the facts. Headache 51:409-417

7. Goadsby PJ (2009) The vascular theory of migraine--a great story wrecked by the facts. Brain 132:6-7

8. Asghar MS, Hansen AE, Amin FM et al (2011) Evidence for a vascular factor in migraine. Ann Neurol 69:635-645

9. Amin FM, Asghar MS, Hougaard A et al (2013) Magnetic resonance angiography of intracranial and extracranial arteries in patients with spontaneous migraine without aura: a cross-sectional study. Lancet Neurol 12:454-461

10. Hay KM (1979) Pain thresholds in migraine. Practitioner 222:827-833

11. Olesen J (1978) Some clinical features of the acute migraine attack. An analysis of 750 patients. Headache 18:268-271

12. Olesen J (1991) Clinical and pathophysiological observations in migraine and tension-type headache explained by integration of vascular, supraspinal and myofascial inputs. Pain 46:125-132

13. GBD 2015 Neurological Disorders Collaborator Group (2017) Global, regional, and national burden of neurological disorders during 1990-2015: a systematic analysis for the Global Burden of Disease Study 2015. Lancet Neurol 16:877-897

14. Lipchik GL, Holroyd KA, O'Donnell FJ et al (2000) Exteroceptive suppression periods and pericranial muscle tenderness in chronic tension-type headache: effects of psychopathology, chronicity and disability. Cephalalgia 20:638-646

15. Buchgreitz L, Lyngberg AC, Bendtsen $L$ et al (2006) Frequency of headache is related to sensitization: a population study. Pain 123:19-27

16. Fernández-de-Las-Peñas C, Cuadrado ML, Arendt-Nielsen L et al (2007) Increased pericranial tenderness, decreased pressure pain threshold, and headache clinical parameters in chronic tension-type headache patients. Clin J Pain 23:346-352

17. Sakai F, Ebihara S, Akiyama M et al (1995) Pericranial muscle hardness in tension-type headache. A non-invasive measurement method and its clinical application. Brain 118(Pt 2):523-531

18. Ashina M, Bendtsen L, Jensen R et al (1999) Muscle hardness in patients with chronic tension-type headache: relation to actual headache state. Pain 79:201-205

19. Simons D, Travell J (1999) Travell \& Simons' myofascial pain and dysfunction: the trigger point manual. Williams \& Wilkins, Baltimore

20. Travell J, Simons D (1952) The myofascial genesis of pain. Postgrad Med 11: $434-452$

21. Stockman R (1904) The causes, pathology and treatment of chronic rheumatism. Edinburgh Med J 15:107-116

22. Shah JP, Thaker N, Heimur J et al (2015) Myofascial trigger point then and now: a historical and scientific prespective. PM R J 7:746-761

23. Wolfe F, Simons D, Fricton J et al (1992) The fibromyalgia and myofascial pain syndromes: a preliminary study of tender points and trigger points in persons with fibromyalgia, myofascial pain syndrome and no disease. J Rheumatol 19:944-951

24. Fernández-De-Las-Peñas C, Dommerholt J Myofascial trigger points: peripheral or central phenomenon? Curr Rheumatol Rep 16. Epub ahead of print 2014. https://doi.org/10.1007/s11926-013-0395-2

25. Shah JP, Danoff JV, Desai MJ et al (2008) Biochemicals associated with pain and inflammation are elevated in sites near to and remote from active myofascial trigger points. Arch Phys Med Rehabil 89:16-23

26. Shah JP, Phillips TM, Danoff JV et al (2005) An in vivo microanalytical technique for measuring the local biochemical milieu of human skeletal muscle. J Appl Physiol 99:1977-1984

27. Sikdar S, Shah JP, Gebreab T et al (2009) Novel applications of ultrasound technology to visualize and characterize myofascial trigger points and surrounding soft tissue. Arch Phys Med Rehabil 90:1829-1838

28. Lewis J, Tehan P (1999) A blinded pilot study investigating the use of diagnostic ultrasound for detecting active myofascial trigger points. Pain 79:39-44

29. Turo D, Otto P, Shah JP et al (2012) Ultrasonic tissue characterization of the upper trapezius muscle in patients with myofascial pain syndrome. Conf Proc IEEE Eng Med Biol Soc 2012:4386-4389

30. Sikdar S, Shah JP, Gilliams E et al (2008) Assessment of myofascial trigger points (MTrPs): a new application of ultrasound imaging and vibration sonoelastography. Conf Proc IEEE Eng Med Biol Soc 2008:5585-5588 
31. Ballyns JJ, Shah JP, Hammond J et al (2011) Objective sonographic measures for characterizing myofascial trigger points associated with cervical pain. J Ultrasound Med 30:1331-1340

32. Takla MKN, Razek NMA, Kattabei $O$ et al (2016) A comparison between different modes of real-time sonoelastography in visualizing myofascial trigger points in low back muscles. J Man Manip Ther 24:253-263

33. Simons DG, Hong C-Z, Simons LS (2002) Endplate potentials are common to midfiber myofacial trigger points. Am J Phys Med Rehabil 81:212-222

34. Ge HY, Monterde S, Graven-Nielsen T et al (2014) Latent myofascial trigger points are associated with an increased intramuscular electromyographic activity during synergistic muscle activation. J Pain 15:181-187

35. Yu SH, Kim HJ (2015) Electrophysiological characteristics according to activity level of myofascial trigger points. J Phys Ther Sci 27:2841-2843

36. Wytrążek M, Huber J, Lipiec J et al (2015) Evaluation of palpation, pressure algometry, and electromyography for monitoring trigger points in young participants. J Manip Physiol Ther 38:232-243

37. Dibai-Filho AV, Guirro RR (2015) Evaluation of myofascial trigger points using infrared thermography: a critical review of the literature. J Manip Physiol Ther 38:86-92

38. Merla A, Ciuffolo F, D'Attilio M et al (2004) Functional infrared imaging in the diagnosis of the myofascial pain. Conf Proc IEEE Eng Med Biol Soc 2:1188-1191

39. Kimura Y, Ge H-Y, Zhang Y et al (2009) Evaluation of sympathetic vasoconstrictor response following nociceptive stimulation of latent myofascial trigger points in humans. Acta Physiol (Oxf) 196:411-417

40. Zhang Y, Ge H-Y, Yue S-W et al (2009) Attenuated skin blood flow response to nociceptive stimulation of latent myofascial trigger points. Arch Phys Med Rehabil 90:325-332

41. Haddad DS, Brioschi ML, Arita ES (2012) Thermographic and clinical correlation of myofascial trigger points in the masticatory muscles. Dentomaxillofac Radiol 41:621-629

42. Chen Q, Wang H, Gay RE et al (2016) Quantification of myofascial taut bands. Arch Phys Med Rehabil 97:67-73

43. Eller-Smith OC, Nicol AL, Christianson JA (2018) Potential mechanisms underlying centralized pain and emerging therapeutic interventions. Front Cell Neurosci 12:35

44. Tfelt-Hansen P, Lous I, Olesen J (1981) Prevalence and significance of muscle tenderness during common migraine attacks. Headache 21:49-54

45. Calandre EP, Hidalgo J, García-Leiva JM et al (2006) Trigger point evaluation in migraine patients: an indication of peripheral sensitization linked to migraine predisposition? Eur J Neurol 13:244-249

46. Fernández-de-Las-Peñas C, Cuadrado ML, Pareja JA (2006) Myofascial trigger points, neck mobility and forward head posture in unilateral migraine. Cephalalgia 26:1061-1070

47. Ferracini GN, Florencio LL, Dach F et al (2017) Myofascial trigger points and migraine-related disability in women with episodic and chronic migraine. Clin J Pain 33:109-115

48. Ferracini GN, Chaves TC, Dach F et al (2016) Relationship between active trigger points and head/neck posture in patients with migraine. Am J Phys Med Rehabil. 95:831-839

49. Florencio LL, Ferracini GN, Chaves TC et al (2017) Active trigger points in the cervical musculature determine the altered activation of superficial neck and extensor muscles in women with migraine. Clin J Pain 33:238-245

50. Gandolfi M, Geroin C, Valè N et al Does myofascial and trigger point treatment reduce pain and analgesic intake in patients undergoing OnabotulinumtoxinA injection due to chronic intractable migraine? A pilot, single-blind randomized controlled trial. Eur J Phys Rehabil Med. Epub ahead of print 27 July 2017. https://doi.org/10.23736/S1973-9087.17.04568-3

51. Ghanbari A, Askarzadeh S, Petramfar P et al (2015) Migraine responds better to a combination of medical therapy and trigger point management than routine medical therapy alone. NeuroRehabilitation 37:157-163

52. Giamberardino MA, Tafuri E, Savini A et al (2007) Contribution of myofascial trigger points to migraine symptoms. J Pain 8:869-878

53. Landgraf MN, Biebl JT, Langhagen T et al Children with migraine: provocation of headache via pressure to myofascial trigger points in the trapezius muscle? - a prospective controlled observational study. Eur J Pain. Epub ahead of print 26 September 2017. https://doi.org/10.1002/ejp.1127

54. Landgraf MN, Ertl-Wagner B, Koerte IK et al (2015) Alterations in the trapezius muscle in young patients with migraine--a pilot case series with MRI. Eur J Paediatr Neurol 19:372-376
55. Palacios-Ceña M, Ferracini GN, Florencio LL et al (2017) The number of active but not latent trigger points associated with widespread pressure pain hypersensitivity in women with episodic migraines. Pain Med 18: 2485-2491

56. Ranoux D, Martiné G, Espagne-Dubreuilh G et al (2017) OnabotulinumtoxinA injections in chronic migraine, targeted to sites of pericranial myofascial pain: an observational, open label, real-life cohort study. J Headache Pain. 18:75

57. Sollmann N, Trepte-Freisleder F, Albers L et al (2016) Magnetic stimulation of the upper trapezius muscles in patients with migraine - a pilot study. Eur J Paediatr Neurol 20:888-897

58. Tali D, Menahem I, Vered E et al (2014) Upper cervical mobility, posture and myofascial trigger points in subjects with episodic migraine: case-control study. J Bodyw Mov Ther 18:569-575

59. Affaitati G, Martelletti P, Lopopolo M et al (2017) Use of nonsteroidal antiinflammatory drugs for symptomatic treatment of episodic headache. Pain Pract 17:392-401

60. Jensen RH (2017) Tension-type headache - the normal and most prevalent headache. Headache:1-7

61. Kolding LT, Do TP, Ewertsen C et al (2018) Muscle stiffness in tension-type headache patients with pericranial tenderness. Cephalalgia Reports 1: 251581631876029

62. Alonso-Blanco C, Fernández-de-las-Peñas C, Fernández-Mayoralas DM et al (2011) Prevalence and anatomical localization of muscle referred pain from active trigger points in head and neck musculature in adults and children with chronic tension-type headache. Pain Med 12:1453-1463

63. Fernández-de-las-Peñas C, Fernández-Mayoralas DM, Ortega-Santiago R et al (2011) Referred pain from myofascial trigger points in head and neckshoulder muscles reproduces head pain features in children with chronic tension type headache. J Headache Pain. 12:35-43

64. Fernández-de-las-Peñas C, Cuadrado ML, Gerwin RD et al (2005) Referred pain from the trochlear region in tension-type headache: a myofascial trigger point from the superior oblique muscle. Headache 45:731-737

65. Fernández-de-las-Peñas C, Alonso-Blanco C, Cuadrado ML et al (2006) Trigger points in the suboccipital muscles and forward head posture in tension-type headache. Headache 46:454-460

66. Fernández-de-Las-Peñas C, Ge H-Y, Arendt-Nielsen L et al (2007) The local and referred pain from myofascial trigger points in the temporalis muscle contributes to pain profile in chronic tension-type headache. Clin J Pain 23:786-792

67. Couppé C, Torelli P, Fuglsang-Frederiksen A et al (2007) Myofascial trigger points are very prevalent in patients with chronic tension-type headache: a double-blinded controlled study. Clin J Pain 23:23-27

68. Fernández-de-las-Peñas C, Caminero AB, Madeleine P et al (2009) Multiple active myofascial trigger points and pressure pain sensitivity maps in the temporalis muscle are related in women with chronic tension type headache. Clin J Pain 25:506-512

69. Fernández-de-Las-Peñas C, Cuadrado ML, Pareja JA (2007) Myofascial trigger points, neck mobility, and forward head posture in episodic tension-type headache. Headache 47:662-672

70. Fernández-de-Las-Peñas C, Ge H-Y, Arendt-Nielsen L et al (2007) Referred pain from trapezius muscle trigger points shares similar characteristics with chronic tension type headache. Eur J Pain 11:475-482

71. Fernández-de-Las-Peñas C, Alonso-Blanco C, Cuadrado ML et al (2006) Myofascial trigger points in the suboccipital muscles in episodic tensiontype headache. Man Ther 11:225-230

72. Fernández-de-Las-Peñas C, Alonso-Blanco C, Cuadrado ML et al (2006) Myofascial trigger points and their relationship to headache clinical parameters in chronic tension-type headache. Headache 46:1264-1272

73. Harden RN, Cottrill J, Gagnon CM et al (2009) Botulinum toxin a in the treatment of chronic tension-type headache with cervical myofascial trigger points: a randomized, double-blind, placebo-controlled pilot study. Headache 49:732-743

74. Karadaş Ö, Inan LE, Ulaş ÜH et al (2013) Efficacy of local lidocaine application on anxiety and depression and its curative effect on patients with chronic tension-type headache. Eur Neurol 70:95-101

75. Lattes K, Venegas $P$, Lagos $N$ et al (2009) Local infiltration of gonyautoxin is safe and effective in treatment of chronic tension-type headache. Neurol Res 31:228-233

76. Moraska AF, Schmiege SJ, Mann JD et al (2017) Responsiveness of myofascial trigger points to single and multiple trigger point release massages: a randomized, placebo controlled trial. Am J Phys Med Rehabil. 96:639-645 
77. Moraska AF, Stenerson L, Butryn N et al (2015) Myofascial trigger pointfocused head and neck massage for recurrent tension-type headache: a randomized, placebo-controlled clinical trial. Clin J Pain 31:159-168

78. Palacios-Ceña $\mathrm{M}$, Wang $\mathrm{K}$, Castaldo $\mathrm{M}$, et al. Trigger points are associated with widespread pressure pain sensitivity in people with tension-type headache. Cephalalgia. Epub ahead of print 14 November 2016. https://doi. org/10.1177/0333102416679965

79. Romero-Morales C, Jaén-Crespo G, Rodríguez-Sanz D et al (2017) Comparison of pressure pain thresholds in upper trapezius and temporalis muscles trigger points between tension type headache and healthy participants: a case-control study. J Manip Physiol Ther 40:609-614

80. Sohn J, Choi H, Lee S-M et al (2010) Differences in cervical musculoskeletal impairment between episodic and chronic tension-type headache. Cephalalgia 30:1514-1523

81. Karadaş Ö, Gül HL, Inan LE (2013) Lidocaine injection of pericrania myofascial trigger points in the treatment of frequent episodic tension-type headache. J Headache Pain 14:44

82. Schytz HW, Amin FM, Selb J et al (2017) Non-invasive methods for measuring vascular changes in neurovascular headaches. J Cereb Blood Flow Metab 271678X17724138

83. Ashina M, Stallknecht B, Bendtsen L et al (2003) Tender points are not sites of ongoing inflammation - In vivo evidence in patients with chronic tension-type headache. Cephalalgia 23:109-116

84. Giamberardino MA, Affaitati G, Martelletti P et al (2015) Impact of migraine on fibromyalgia symptoms. J Headache Pain. 17:28

85. Wolfe F, Smythe HA, Yunus MB et al (1990) The American College of Rheumatology 1990 criteria for the classification of fibromyalgia. Report of the multicenter criteria committee. Arthritis Rheum 33:160-172

86. Ifergane $G$, Buskila D, Simiseshvely $N$ et al (2006) Prevalence of fibromyalgia syndrome in migraine patients. Cephalalgia 26:451-456

87. Jackson JL, Kuriyama A, Hayashino Y (2012) Botulinum toxin a for prophylactic treatment of migraine and tension headaches in adults: a meta-analysis. JAMA 307:1736-1745

88. Do TP, Hvedstrup J, Schytz HW (2018) Botulinum toxin: a review of the mode of action in migraine. Acta Neurol Scand 137:442-451

89. Gerwin RD, Shannon S, Hong CZ et al (1997) Interrater reliability in myofascial trigger point examination. Pain 69:65-73

Ready to submit your research? Choose BMC and benefit from:

- fast, convenient online submission

- thorough peer review by experienced researchers in your field

- rapid publication on acceptance

- support for research data, including large and complex data types

- gold Open Access which fosters wider collaboration and increased citations

- maximum visibility for your research: over $100 \mathrm{M}$ website views per year

At $\mathrm{BMC}$, research is always in progress.

Learn more biomedcentral.com/submissions 